\title{
Prognostic value of myocardial fibrosis in severe aortic stenosis: study protocol for a prospective observational multi-center study (FIB-AS)
}

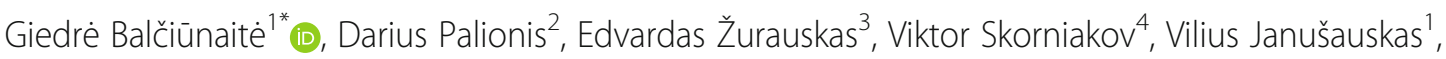
Aleksejus Zorinas ${ }^{1}$, Tomas Zaremba ${ }^{1,5}$, Nomeda Valevičiené ${ }^{2}$, Audrius Aidietis ${ }^{1}$, Pranas Šerpytis ${ }^{1}$, Kęstutis Ručinskas $^{1}$, Peter Sogaard ${ }^{1,5}$ and Sigita Glaveckaite ${ }^{1}$

\begin{abstract}
Background: Adverse cardiac remodeling with a myocardial fibrosis as a key pathophysiologic component may be associated to worse survival in aortic stenosis (AS) patients. Therefore, with the application of advanced cardiac imaging we aim to investigate left ventricular myocardial fibrosis in severe AS patients undergoing aortic valve replacement (AVR) and determine its impact with post-intervention clinical outcomes.

Methods: In a prospective, observational, cohort study patients with severe AS scheduled either for surgical or transcatheter AVR will be recruited from two tertiary heart centers in Denmark and Lithuania. All patients will receive standard of care in accordance with the current guidelines and will undergo additional imaging testing before and after AVR: echocardiography with deformation analysis and cardiovascular magnetic resonance (CMR) with T1 parametric mapping. Those undergoing surgical AVR will also have a myocardial biopsy sampled at the time of a surgery for histological validation. Patients will be recruited over a 2-year period and followed up to 2 years to ascertain clinical outcomes. Follow-up CMR will be performed 12 months following AVR, and echocardiography with deformation analysis will be performed 3, 12, and 24 months following AVR. The study primary outcome is a composite of all-cause mortality and major adverse cardiovascular events.
\end{abstract}

Discussion: Despite continuous effort of research community there is still a lack of early predictors of left ventricular decompensation in AS, which could improve patient risk stratification and guide the optimal timing for aortic valve intervention, before irreversible left ventricular damage occurs. Advanced cardiac imaging and CMR derived markers of diffuse myocardial fibrosis could be utilized for this purpose. FIB-AS study is intended to invasively and non-invasively assess diffuse myocardial fibrosis in AS patients and investigate its prognostic significance in post-interventional outcomes. The results of the study will expand the current knowledge of cardiac remodeling in AS and will bring additional data on myocardial fibrosis and its clinical implications following AVR.

\footnotetext{
* Correspondence: dr.giedre.balciunaite@gmail.com

'Clinic of Cardiac and Vascular Diseases, Institute of Clinical Medicine, Vilnius University Faculty of Medicine, Santariškiu str. 2, LT-08661 Vilnius, Lithuania

Full list of author information is available at the end of the article
}

(c) The Author(s). 2020 Open Access This article is licensed under a Creative Commons Attribution 4.0 International License, which permits use, sharing, adaptation, distribution and reproduction in any medium or format, as long as you give appropriate credit to the original author(s) and the source, provide a link to the Creative Commons licence, and indicate if changes were made. The images or other third party material in this article are included in the article's Creative Commons licence, unless indicated otherwise in a credit line to the material. If material is not included in the article's Creative Commons licence and your intended use is not permitted by statutory regulation or exceeds the permitted use, you will need to obtain permission directly from the copyright holder. To view a copy of this licence, visit http://creativecommons.org/licenses/by/4.0/ The Creative Commons Public Domain Dedication waiver (http://creativecommons.org/publicdomain/zero/1.0/) applies to the data made available in this article, unless otherwise stated in a credit line to the data. 
(Continued from previous page)

Ethics/dissemination: The study has full ethical approval and is actively recruiting patients. The results will be disseminated through scientific journals and conference presentations.

Trial registration: ClinicalTrials.gov NCT03585933. Registered on 02 July 2018.

Keywords: Magnetic resonance imaging, Aortic valve stenosis, Myocardial fibrosis, Aortic valve replacement, Prognosis

\section{Background}

Since increased afterload is the main pathophysiology of aortic stenosis (AS) leading to the progressive development of left ventricular (LV) structural and functional alterations, AS is regarded a disease of the aortic valve, as well as of the myocardium [1]. The increasing wall stress triggers cardiac fibroblasts to upregulate fibronectin synthesis, with subsequent alteration in collagen architecture and myocardial fibrosis, compromising systolic and diastolic LV function [2]. Two distinct types of myocardial fibrosis have been described: (i) diffuse myocardial fibrosis and (ii) focal replacement fibrosis [3]. Focal replacement fibrosis can be detected by cardiovascular magnetic resonance (CMR) with late gadolinium enhancement (LGE) and is observed in up to half of aortic stenosis patients with non-infarct type being the most prevalent [4]. Multicenter trials and meta-analyses have shown that the presence and extent of LGE are associated with higher long-term all-cause and cardiovascular mortality after aortic valve replacement (AVR), indicating more advanced myocardial injury $[4,5]$. Focal myocardial fibrosis has been demonstrated to be irreversible following AVR $[6,7]$, resulting in incomplete LV recovery and worse clinical outcomes, suggesting suboptimal timing of aortic valve intervention in some patients. On the contrary, diffuse interstitial fibrosis has been reported to be reversible with afterload relief and could be utilized as a potential marker of early LV dysfunction [8, 9]. However, the most recent study showed that regression of diffuse fibrosis may be incomplete in certain patients, leading to persistent LV systolic dysfunction and worse survival [10]. Application of T1 mapping techniques, which measures native and postcontrast T1 relaxation time or extracellular volume fraction (ECV), facilitates non-invasive detection and quantification of interstitial fibrosis with high spatial resolution [11-14]. Several studies in AS patients have reported that native $\mathrm{T} 1$ and ECV values correlate with the degree of diffuse myocardial fibrosis and predict cardiovascular events and mortality $[9,10,15]$. Thus, myocardial fibrosis detection by CMR is potentially useful for improving patient risk stratification and perhaps can justify earlier aortic valve intervention, before extensive fibrosis and irreversible myocardial damage develop.

Furthermore, because the subendocardial layer is the first to be affected in AS, the longitudinal alignment of myocardial fibers in this layer causes decreased longitudinal contraction, an early sign of LV dysfunction. There are data showing, that global longitudinal strain (GLS) correlate with LV myocardial fibrosis and is a predictor of adverse events in patients with severe AS [16, 17].

Novel diagnostic strategies and more accurate evaluation of the disease severity and consequences of AS are needed in the assessment of subclinical myocardial dysfunction and the detection of myocardial fibrosis to challenge current recommendations for the timing of AVR. To date there are limited data on simultaneous assessment of diffuse myocardial fibrosis by noninvasive multimodality imaging and histological confirmation in severe AS. Optimal T1 mapping application also remains unclear, and data assessing its prognostic value in severe AS are still lacking. Our multicenter prospective trial aims to: (i) non-invasively assess myocardial fibrosis and validate it against histological data in patients undergoing surgical AVR and (ii) assess the impact of myocardial fibrosis on clinical outcomes following AVR in both surgical and TAVR cohorts.

\section{Hypothesis}

Primary hypothesis: diffuse and focal myocardial fibrosis in patients with severe AS is associated with worse immediate (in-hospital) and long-term clinical outcomes, all-cause mortality, and major adverse cardiovascular events (MACEs).

Secondary hypotheses:

- The presence and extent of myocardial fibrosis are associated with markers of LV decompensation.

- The presence and extent of myocardial fibrosis have a negative effect on LV reverse remodeling and patient functional recovery following AVR.

\section{Study objectives}

Primary objective:

- To evaluate the prognostic significance of myocardial fibrosis in patients with severe AS undergoing AVR. 
Secondary objectives:

- To identify parameters of multimodality imaging [two-dimensional (2D) echocardiography with an extended myocardial deformation analysis, $1.5 \mathrm{~T}$ contrast-enhanced CMR with T1 parametric mapping] predictive of LV decompensation.

- To quantify LV reverse remodeling 12 months following AVR through CMR and echocardiographic measurements.

\section{Methods}

\section{Study design}

FIB-AS is a prospective, observational, cohort study with clinical endpoints, conducted in two participating sites (in Lithuania and Denmark) from tertiary care hospitals. A total of 110 patients with severe AS undergoing AVR will be recruited from both institutions, and their data will be collected in a dedicated online database, REDCap (Research Electronic Data Capture) [18]. The choice of aortic valve intervention, either surgical or transcatheter, will be based on the heart team's decision in accordance to current guidelines [19]. Standard work-up examinations for surgical AVR or TAVR will be conducted, consisting of coronary angiography, echocardiography, computed tomography, electrocardiography, and blood tests. Once consent is confirmed, and prior to the AVR procedure (window of $0-30$ days), a contrast-enhanced CMR scan with T1 mapping will be performed, in addition to the standard-of-care. A follow-up CMR scan will be performed 1 year following AVR. Those undergoing surgical AVR will also have a myocardial biopsy sampled at the time of a surgery, which will be sent for histological evaluation. Outcome assessments will continue for a total of 2 years post-intervention. The study flow diagram is presented in Fig. 1. Both sites have received approval from local ethics committees (Approval Numbers: for Vilnius University Hospital (VUH): 158200-18/9-1014-558; for Aalborg University Hospital (AAUH): N-20180081. All patients will give written informed consent.

\section{Study population}

We will recruit patients with severe AS requiring AVR according to current treatment recommendations [19]. We will exclude patients with obstructive coronary artery disease requiring revascularization or other significant valvular pathology. The inclusion and exclusion criteria are set in Table 1 . The recruitment period will be 24 months, from May 2019 to May 2021. We expect

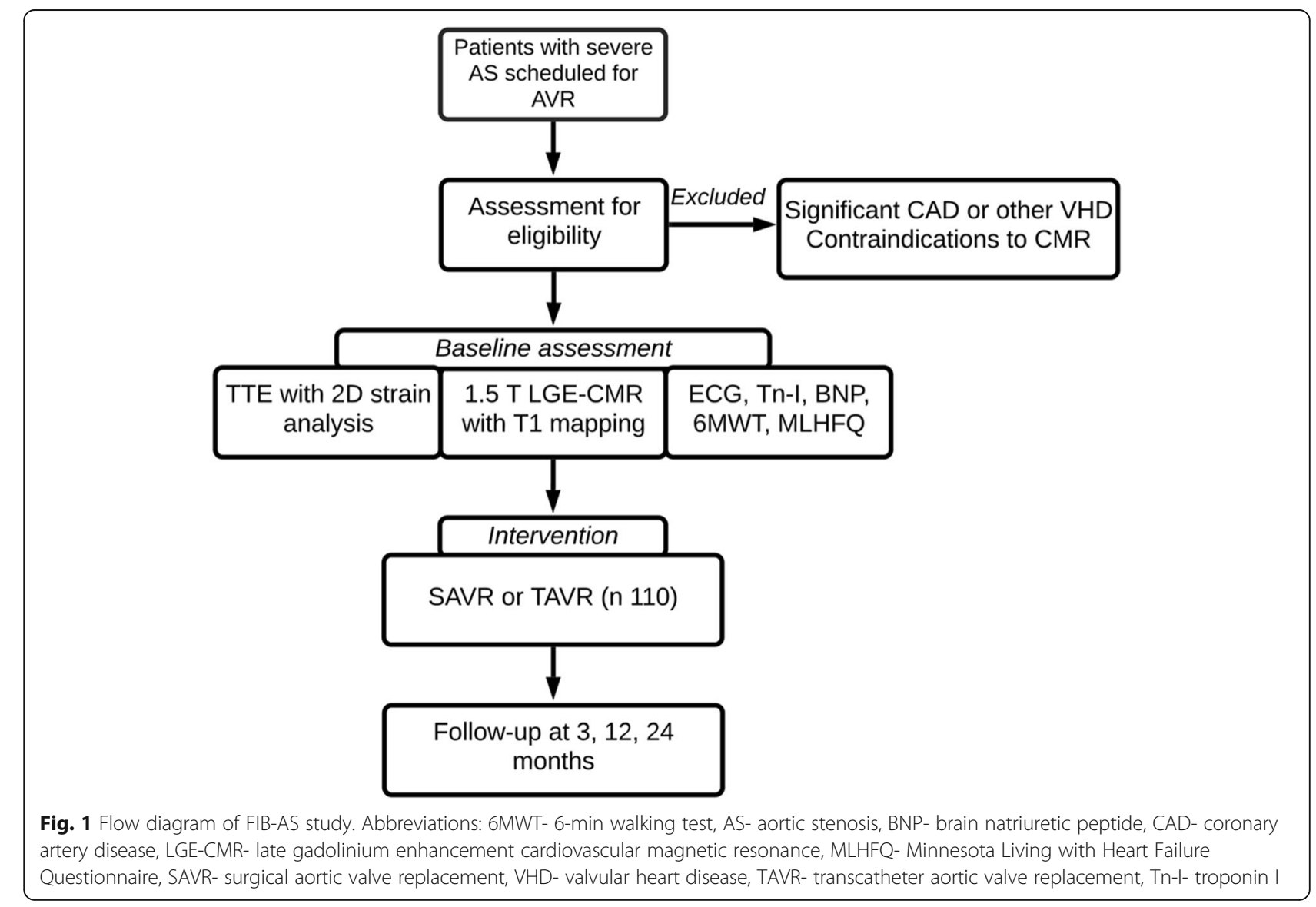


Table 1 Inclusion and exclusion criteria

Inclusion Criteria:
- Severe AS (defined as AVA $\leq 1 \mathrm{~cm}^{2}$ or AVA index (iAVA) $\leq 0.6 \mathrm{~cm}^{2} / \mathrm{m}^{2}$
as determined by ultrasound examination)
- Males and females of any ethnic group $\geq 18$ years of age
- Signed an informed patient consent form
Exclusion Criteria:
- Unable to provide informed consent
- Severe valvular heart disease other than AS
- Coronary artery disease requiring revascularization
- History of myocardial infarction
- Prior cardiac surgery
- Severe renal impairment - eGFR < $30 \mathrm{ml} /$ min/1.73 m²
- Any absolute contraindication to CMR
- Permanent atrial fibrillation
- Patient with implanted cardiac devices (pacemaker, ICD)
- Inherited or acquired cardiomyopathy
- Other medical conditions that limit life expectancy or preclude AVR
- Pregnant or nursing women
- Mental condition rendering the patient unable to understand the
nature, scope, and possible consequences of the study or to follow the
protocol

Abbreviations: AS- aortic stenosis, AVA- aortic valve area, iAVA- aortic valve area index, AVR- aortic valve replacement, CMR- cardiovascular magnetic resonance, eGFR- estimated glomerular filtration rate, ICD- implantable cardioverter defibrillator

to enroll a total of 110 patients: 60 patients undergoing surgical AVR and 50 patients undergoing TAVR.

\section{Aortic valve replacement}

Surgical AVR will be performed by standard midline sternotomy or J mini sternotomy with cardiopulmonary bypass and mild hypothermia. The St. Jude Medical Trifecta aortic bioprosthesis (St. Jude Medical, Inc., St. Paul, MN, USA) or mechanical CarboMedics Standard Aortic Valve (CarboMedics, Inc.; Austin, Tex) prostheses of varying sizes will be used according to the surgical team's or patient's preference. TAVR will be performed under conscious sedation. A balloon-expandable Edwards Sapien-3 Ultra Valve system, size 23, 26, or 29 mm (Edwards Lifesciences Inc., Irvine, CA, USA), will be deployed. Preferably a transfemoral route of access will be used, when vascular access will be judged suitable.

\section{Data collection}

The risk of interventional mortality will be assessed by calculating European System for Cardiac Operative Risk Evaluation II (EuroSCORE II) risk scores and Society of Thoracic Surgeons (STS) risk scores using the online calculators available at http://www.euroscore.org/calc.html [20] and at http://riskcalc.sts.org/stswebriskcalc [21]. Although not designed for TAVR, the STS score has been validated and proved as a sensitive predictor of 30-day mortality and may be used in both SAVR and TAVR groups [22]. New York Heart Association (NYHA) functional class and 6-min walking test (6MWT) will be applied to assess patients' functional capacity and performed using standard method [23]. The Minnesota Living with Heart Failure Questionnaire (MLHFQ) [24] will be used to assess health-related quality of life from the patient's perspective, as it has been specifically validated in patients with aortic valve disease [25].

\section{Blood testing}

Blood samples will be collected and levels of cardiac biomarkers (B-type natriuretic peptide and troponin I) will be tested at baseline and at each follow-up visit. In addition, blood samples will be taken on the day of CMR scanning to determine hematocrit and creatinine concentration.

\section{Echocardiography}

Comprehensive transthoracic 2D echocardiography will be performed using a commercially available Vivid ultrasound (S70, E9 or E95) systems (GE Healthcare, Horten, Norway) and stored on a dedicated workstation for subsequent off-line analysis. AS severity, LV systolic and diastolic functions will be evaluated in accordance with the echocardiographic guidelines [26-28]. Simpson's biplane method will be used to calculate LV ejection fraction. The aortic valve area at the pre-interventional assessment and the effective prosthetic orifice area in the post-interventional assessment will be calculated using the continuity eq.

\section{$2 D$ speckle tracking echocardiography (STE)}

From the 2D grey-scale images of the apical two-, three-, and four-chamber views, left ventricular global longitudinal strain (GLS) will be measured and processed offline using commercially available software (EchoPac 112.0.1, GE Medical Systems; Horten, Norway) [29]. The frame rate will be adjusted to 50 to 80 frames/s. The end-systole will be defined by detecting the closure click on the spectral tracing of the pulsed-wave Doppler of aortic valve flow. During analysis, the endocardial border will be traced manually at an end-systolic frame, and the software will automatically trace a region of interest that includes the entire myocardium. The change in length versus the initial length of the speckle pattern over the cardiac cycle will be used to calculate longitudinal strain, with myocardial lengthening or stretching represented as positive strain and myocardial shortening defined as negative strain. GLS will be acquired by the average regional strain curves (16-segment model for 2D STE). 2D STE analysis will be performed only in patients from VUH by an accredited investigator (G.B.). Segments with 
poor quality tracking or aberrant curves despite manual adjustment will be removed from analysis.

\section{CMR protocol Basic description}

For each patient, baseline and 1-year post-interventional CMR scans will be performed using standard protocols on a $1.5 \mathrm{~T}$ Siemens Aera scanner (at VUH) or a 1.5 T GE Discovery MR 450 scanner (at AAUH) with surface coils and prospective ECG triggering [3, 9]. Both sites will use corresponding CMR protocols. LV end-systolic and enddiastolic diameters, as well as maximal (end-diastolic) wall thickness, will be traced and recorded from the short-axis and long-axis views of the standard ECG-gated steady state-free precession cine sequence (8-mm slice thickness). LV volumes, mass, and ejection fraction will be measured using commercially available software (suiteHEART ${ }^{\bullet}$, NeoSoft, USA or cmr42, Circle Cardiovascular Imaging Inc., Canada) from a stack of sequential 8-mm short axis slices (0-2-mm gap) from the atrio-ventricular ring to the apex. Measurements will be indexed to body surface area in $\mathrm{m}^{2}$ (using the DuBois and DuBois formula).

\section{LGE imaging}

For detection of delayed hyperenhancement, images will be acquired $10-15 \mathrm{~min}$ after intravenous administration of Gadobutrol $(0.2 \mathrm{mmol} / \mathrm{kg})$ (Gadovist; Bayer AG, Germany) or Gadoteridol $(0.2 \mathrm{mmol} / \mathrm{kg})$ (Prohance; Bracco Imaging, Sweden) using a breath-hold segmented inversion recovery fast gradient echo sequence in the short-axis and long-axis planes of the LV, with an 8-mm slice thickness and $20 \%$ distance factor. The image parameters (VUH) are: repetition time of $700 \mathrm{~ms}$, echo time $1.42 \mathrm{~ms}$, flip angle $45^{\circ}$, matrix $256 \times 184$, and field of view $360 \mathrm{~mm}$. For AAUH, the typical imaging parameters are as follows: repetition time $5.79 \mathrm{~ms}$, echo time $2.72 \mathrm{~ms}$, flip angle $15^{\circ}$, matrix $256 \times 256$, and field of view $380 \mathrm{~mm}$. The optimal inversion time will be optimized for each patient to null normal myocardial signal, ranging from 220 to $320 \mathrm{~ms}$ (usually around $240 \mathrm{~ms}$ ). The region of myocardial fibrosis will be defined as the sum of pixels with signal intensity above 5 standard deviations of the normal remote myocardium in each short-axis slice. The presence of myocardial fibrosis will be qualitatively determined by two independent readers, blinded to clinical data ( 2 radiologists with $>10$ years of experience at VUH- N.V. and D.P., and 2 cardiologists with $8-10$ years of experience at AAUH- P.S. and T.Z.).

\section{T1 mapping}

The present study describes image acquisition parameters by focusing on T1 mapping images, which will be acquired in four-chamber long-axis and short-axis (at the basal and midventricular levels) images before and at
15 min after contrast administration. All T1 mapping images have been acquired using modified Look-Locker inversion-recovery (MOLLI) $[30,31]$ using the Motion Correction technique (on the Siemens scanner). The following readout parameters will be used: slice thickness: $8 \mathrm{~mm}$; flip angle: 35; field of view: $384 \times 327$; effective TI: $193 \mathrm{~ms}$; voxel size: $1.5 \times 1.5 \times 8 \mathrm{~mm}$; TR/TE: $312.64 / 1.33 \mathrm{~ms}$; partial Fourier: 7/8; and parallel imaging factor: 2 (VUH) and slice thickness $8 \mathrm{~mm}$, flip angle 35, matrix size $256 \times 256$, inversion time $130 \mathrm{~ms}$, and FOV $380 \mathrm{~mm}(\mathrm{AAUH})$.

\section{Measurement of the ECV and native $T 1$ values}

The T1 maps will be generated from the CMR workstation after in-line motion correction just after image acquisition (Siemens scanner) or in post-processing software (GE scanner). Two blinded reviewers will independently review all T1 mapping sequences. Regions of interest will be drawn manually in the blood and septum at the midventricular level on the short-axis image, excluding the myocardium with LGE for T1 measurements [13, 32]. Regions of interest in the septum are chosen for myocardial T1, because this area corresponds to the site of myocardial biopsy. The regions of interest will be drawn on the compact myocardium, and the border of the myocardium will not be included. To measure the T1 value of blood, circular regions of interest will be positioned in the LV cavity, avoiding papillary muscle (Fig. 2).

The ECV of the myocardium will be calculated as follows: $\mathrm{ECV} \%=(\Delta \mathrm{R} 1 \mathrm{~m} / \Delta \mathrm{R} 1 \mathrm{~b}) \times(1-$ hematocrit level $) \times$ 100 , where $\mathrm{R} 1$ is $1 / \mathrm{T} 1, \mathrm{R} 1 \mathrm{~m}$ is $\mathrm{R} 1$ in the myocardium, $\mathrm{R} 1 \mathrm{~b}$ is $\mathrm{R} 1$ in the blood, and $\Delta \mathrm{R} 1$ is the change in relaxivity. The change in relaxivity $(\Delta \mathrm{R} 1)$ was determined using the following equation: $\Delta \mathrm{R} 1=\mathrm{R} 1$ post $-\mathrm{R} 1$ pre, where R1post and R1pre are the R1 after and before gadolinium chelate administration, respectively [33]. Blood samples will be taken on the day of CMR scanning to determine hematocrit and creatinine concentration. Interobserver agreement between 2 different experienced observers will be assessed on different days for 10 randomly selected patients. Figure 3 summarizes the CMR sequences used in this study.

\section{Histological analysis}

In patients undergoing surgical AVR biopsy specimens will be obtained under direct vision by the surgical team using a surgical scalpel from the basal anteroseptum just after removal of the diseased aortic valve. All myocardium tissue samples will be fixed in 10\% neutral buffered formalin and embedded in paraffin. Sections of 3- $\mu \mathrm{m}$ thickness will be made on a Leica RM2145 microtome and stained by hematoxylin and eosin, Masson's trichrome, and Congo red methods. Digital images will be captured by using the Aperio Scan-Scope XT Slide Scanner (Aperio Technologies, Vista, CA, USA) under $20 \times$ objective magnification 


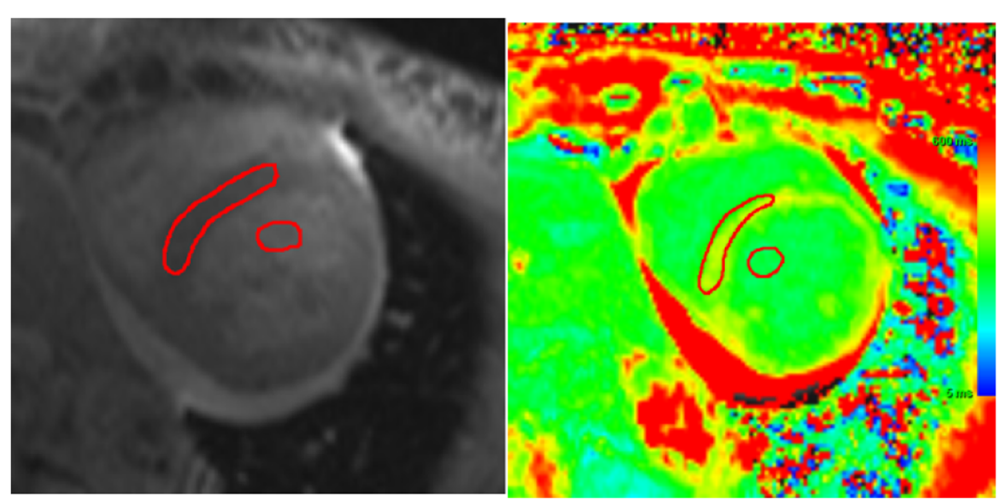

Fig. 2 CMR T1 assessment. To measure the T1 value, regions of interest will be drawn manually in the blood and septum at the midventricular level on the short-axis, avoiding papillary muscle

(0.5- $\mu \mathrm{m}$ resolution). Histological slides (typically measuring 20-30 sq. $/ \mathrm{mm}$ ) will be processed for evaluation of myocardial fibrosis. An experienced ( $>25$ years of experience) histologist (E.Ž.) blinded to the clinical and CMR data will investigate all biopsy specimens. The fraction of myocardial volume occupied by collagen tissue (collagen volume fraction) will be determined by quantitative morphometry with an automated image analysis system $\left(\mathrm{HALO}^{\mathrm{rm}}\right)$ in sections stained with Masson's trichrome. Quantification of the myocardial fibrosis area will be performed using $\mathrm{HALO}^{\text {ma }}$ Classifier Module and $\mathrm{HALO}^{\mathrm{max}}$ Area Quantification v1.0 algorithms (IndicaLabs, NM, USA) within manually selected regions of interest enclosing the tissue section and excluding endocardium from the analysis. All study assessments are summarized in Table 2.

\section{Outcome measures}

Primary outcome measure: a composite of all-cause mortality and MACEs (time frame: from 30 days up to 24 months following AVR).

Secondary outcomes measures:
- In-hospital and 30-day all-cause mortality (time frame: 30 days)

- Length of hospital stay

- Time to the event (death or MACE) (time frame: 24 months)

- Cardiovascular mortality (from 30 days up to 24 months following AVR)

Primary outcome endpoints will be defined according to current guidelines: Valve Academic Research Consortium-2 (VARC-2) definitions [34] and guidelines for reporting mortality and morbidity after cardiac valve interventions [35]. Cardiovascular mortality defined as: (1) death due to proximate cardiac cause (e.g., myocardial infarction, cardiac tamponade, worsening heart failure, and low cardiac output syndrome); (2) death caused by non-coronary vascular conditions (e.g., pulmonary embolism, stroke, aortic rupture, or vascular dissection); (3) all procedure-related deaths; (4) all valve-related deaths; and (5) sudden or unwitnessed death [35]. Allcause mortality defined as the sum of cardiovascular and non-cardiovascular deaths, with the latter defined as any
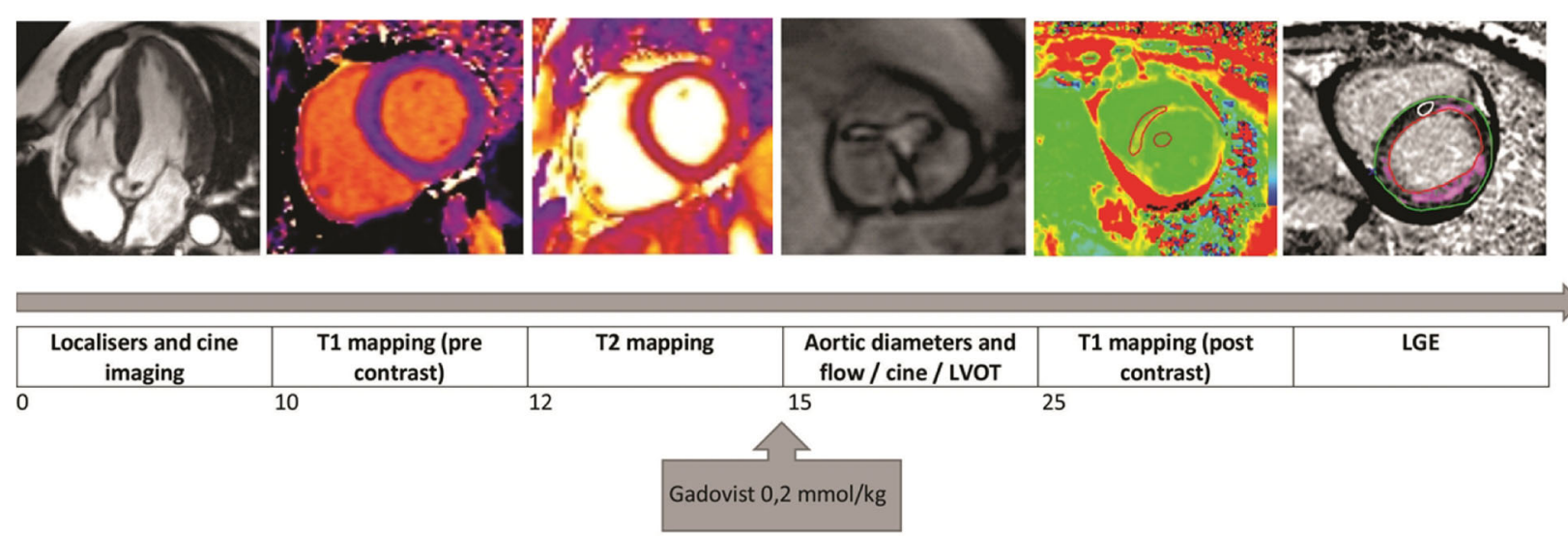

Fig. 3 CMR protocol for FIB-AS study 
Table 2 Study assessments

Demographic data and comorbidities
STS risk score
EuroSCORE II
Serum biomarkers:
- Brain natriuretic peptide
- Troponin I
Cardiovascular imaging biomarkers:
- Transthoracic 2D echocardiogram with strain analysis (GLS)
- 1.5 T contrast-enhanced CMR with T1 mapping (Native T1, ECV)
Myocardial histological analysis:
- Quantitative myocardial fibrosis assessment (CVF)
Functional status and quality of life assessment:
- New York Heart Association functional class
- Minnesota Living with Heart Failure Questionnaire
-6-min walking test

Abbreviations: 2D- two-dimensional, ECV- extracellular volume, EuroSCOREEuropean System for Cardiac Operative Risk Evaluation, CMR- cardiovascular magnetic resonance, CVF- collagen volume fraction, GLS- global longitudinal strain, STS- Society of Thoracic Surgeons

death in which the primary cause is clearly related to other than cardiovascular condition. 30-day mortality defined as death occurring within 30 days or during index procedure hospitalization, if the postoperative length of stay is longer than 30 days.

\section{Clinical follow-up}

Patients will be routinely followed and managed according to available guidelines. Clinical outcome data will be collected from patient clinical visits at 3, 12, and 24 months following AVR. Serum biomarker analysis and 2D echocardiography with STE analysis will be performed at each follow-up visit. A second CMR scan will be performed 1 year after AVR. The 6MWT will be performed, and the MLHF questionnaire will be administered at the 12-month follow-up visit. FIB-AS study follows the standard protocol items: recommendation for interventional trials (SPIRIT) guidelines [36]. The study timeline is presented in Fig. 4.

\section{Statistical analysis}

- Linear regression analysis to model the relationship between the extent of myocardial fibrosis and patient outcomes.

- Univariate and multivariate regression analysis of CMR-derived predictors of adverse clinical events.

- Comparison of clinical, serum, and imaging (CMR and echocardiography) biomarker data between patients with different extents of fibrosis.
The statistical analysis will be performed under the supervision of V.S. at the Institute of Applied Mathematics, Vilnius University Faculty of Mathematics and Informatics. Descriptive statistics will be used to summarize the demographic, clinical, and imaging data characteristics of each patient. Categorical variables will be presented as the frequency and percentage, and continuous variables will be expressed as the mean and standard deviation or median and interquartile range. The $95 \%$ confidence interval will be reported for primary outcomes. Patients will be grouped, depending on the presence and extent of histologically measured myocardial fibrosis, and their clinical and imaging data will be compared for significant differences. Bivariate correlation analysis of the native T1, ECV, and GLS values with the degree of histologically measured myocardial fibrosis will be performed. Pearson or Spearman correlation coefficient will be used as the measure of correlation. To identify independent predictors of the degree of myocardial fibrosis, multivariate stepwise regression analysis will be performed separately for native T1, ECV, and GLS values, along with demographic and clinical data. The composite event will be assessed from a simple Cox model for survival analysis using ECV, native T1, and GLS, and their c-indexes will be compared for how well each marker predicts the clinical outcome. In addition, the Kaplan-Meier method will be used for the cumulative survival analysis with log-rank test to assess statistical differences between the curves of patients with different degree of myocardial fibrosis. A two-tailed $p$-value of $<0.05$ will be considered statistically significant. The statistical analysis will be performed using the $\mathrm{R}$ language and environment for statistical computing (version 3.5.1) [37].

\section{Sample size justification}

A sample size calculation was performed for the primary outcome: a composite of all-cause mortality and MACEs following AVR. To estimate the sample size required for the detection of a hazard ratio for $\mathrm{ECV} \%$ of 1.32 , R package powerSurvEpi was used [38]. Calculations were made assuming that the Cox proportional hazards regression model applies and taking into consideration a correlation of ECV\% with other possible confounding covariates. On the basis of previous studies $[15,17]$ and assuming that the power of the test should be at least 0.8 , a sample size of 100 patients is required. After an adjustment for $10 \%$ patient loss or withdrawal, a total sample size of $110 \mathrm{pa}-$ tients was selected. The selected sample size is comparable to other studies testing the association of myocardial fibrosis with adverse clinical outcomes.

\section{Study timetable}

The ethics application was approved in March 2018. Study enrollment started in May 2019, and recruitment is expected to be completed in May 2021, with a further 


\begin{tabular}{|c|c|c|c|c|c|c|c|c|}
\hline \multirow[b]{3}{*}{ TIMEPOINT } & \multicolumn{8}{|c|}{ STUDY PERIOD } \\
\hline & \multirow{2}{*}{\begin{tabular}{|l|} 
Enrolment \\
0 \\
\end{tabular}} & \multirow{2}{*}{$\begin{array}{l}\text { Allocation } \\
0\end{array}$} & \multicolumn{5}{|c|}{ Post-allocation } & \multirow{2}{*}{\begin{tabular}{|l|} 
Close-out \\
$24 m$
\end{tabular}} \\
\hline & & & \begin{tabular}{|l|} 
Pre- \\
AVR \\
\end{tabular} & $A V R$ & $3 m$ & $12 m$ & $24 m$ & \\
\hline \multicolumn{9}{|l|}{ ENROI } \\
\hline \multirow{3}{*}{$\begin{array}{l}\text { Eligibility screen } \\
\text { Informed consent } \\
\text { Allocation }\end{array}$} & $\mathrm{X}$ & & & & & & & \\
\hline & $\mathrm{X}$ & & & & & & & \\
\hline & & $\mathrm{X}$ & & & & & & \\
\hline \multicolumn{9}{|l|}{ INTERVENTIONS: } \\
\hline \multirow{2}{*}{$\begin{array}{l}\text { CMR } \\
\text { Endomyocardial } \\
\text { biopsy }\end{array}$} & & & $\mathrm{x}$ & & & $\mathrm{X}$ & & \\
\hline & & & & $\mathrm{X}^{*}$ & & & & \\
\hline \multirow{2}{*}{$\begin{array}{l}2 D \text { Echo with strain } \\
\text { analysis }\end{array}$} & & & $\mathrm{x}$ & & $\mathrm{x}$ & $\mathrm{x}$ & $\mathrm{x}$ & \\
\hline & & & $\mathrm{x}$ & & $\mathrm{x}$ & $\mathrm{x}$ & $\mathrm{x}$ & \\
\hline Serum biomarkers & & & $\mathrm{x}$ & & & $\mathrm{x}$ & $\mathrm{x}$ & \\
\hline $\begin{array}{l}\text { 6MWT } \\
\text { MLHFQ }\end{array}$ & & & $\mathrm{X}$ & & & $\mathrm{X}$ & $\mathrm{X}$ & \\
\hline \multirow{2}{*}{$\begin{array}{l}\text { ASSESSMENTS: } \\
\begin{array}{l}\text { Baseline } \\
\text { demographics and } \\
\text { comorbidities }\end{array}\end{array}$} & & & & & & & & \\
\hline & $\mathrm{X}$ & $\mathrm{x}$ & $\mathrm{x}$ & & & & & \\
\hline \multirow[t]{2}{*}{ All-cause mortality } & & & & & & & & \\
\hline & & & & & & & & \\
\hline
\end{tabular}

Fig. 4 Standard protocol items: recommendation for interventional trials (SPIRIT) figure of participant timeline. Abbreviations: 2D- twodimensional, 6MWT- 6-min walking test, CMR- cardiovascular magnetic resonance, MACEs- major adverse cardiovascular events, m- months, MLHFQ- Minnesota Living with Heart Failure Questionnaire. *Endomyocardial biopsy will be performed only in patients undergoing surgical AVR

24 months for follow-up, post-processing, and close-out of the study. The main study paper will be submitted within 6 months of the study close-out.

\section{Dissemination of results}

The study results will be presented to the participating physicians and the general medical community. Following the complete data collection, the manuscript(s) based on the trial results will be submitted to peerreviewed journals. Authorship criteria as defined by the International Committee of Medical Journal Editors will be followed.

\section{Discussion}

FIB-AS is a study intended to investigate the role of multimodality imaging in the assessment of myocardial fibrosis in severe aortic stenosis patients and its prognostic significance in post-interventional outcomes. We sought to assess diffuse myocardial fibrosis by measuring the ECV and native T1 values obtained from CMR with T1 mapping and GLS from STE and to validate these results against the degree of myocardial fibrosis found from the histological examination. In addition, we will explore LV reverse remodeling following aortic valve intervention and its associations with patient clinical recovery. The results of the study will expand the current knowledge of cardiac remodeling in AS and will bring additional data on myocardial fibrosis and its clinical implications following AVR.

\section{Strengths}

- The present prospective multicenter study is designed to explore associations between left ventricular myocardial fibrosis and clinical outcomes in severe AS patients undergoing AVR. It has clearly established aims, inclusion and exclusion criteria, as well as defined methods and endpoints. 
- The non-invasive measurement of myocardial fibrosis by CMR with T1 mapping is validated against invasive histological assessment.

- The trial is restricted to isolated AS, excluding cardiac pathologies which could possibly contribute to myocardial fibrosis burden, such as obstructive coronary heart disease and other significant valvular heart diseases.

- Inclusion of both surgical and TAVR cohorts will allow the investigation of patients with different risk profiles.

\section{Limitations}

- The selected sample size may be inadequate to allow a subgroup analysis.

- Limitations of CMR to establish diffuse and focal fibrosis: no reference regions of normal myocardium due to diffuse fibrosis, arbitrary selection of threshold of signal intensity, overlap of T1 values between normal and diseased myocardium.

- The FIB-AS study excludes patients with comorbidities, such as obstructive coronary artery disease, a history of myocardial infarction, renal failure, and persistent atrial arrhythmias; therefore, our results should not be overgeneralized to a broader AS patient population.

\begin{abstract}
Abbreviations
2D STE: Two-dimensional speckle tracking echocardiography; 6MWT: 6-min walking test; AAUH: Aalborg University hospital; AS: Aortic stenosis; AVR: Aortic valve replacement; CAD: Coronary artery disease; CMR: Cardiovascular magnetic resonance; ECV: Extracellular volume; GLS: Global longitudinal strain; LGE: Late gadolinium enhancement; MOLLI: Modified look-locker inversion-recovery; NYHA: New York heart association; TAVR: Transcatheter aortic valve replacement; VUH: Vilnius University hospital
\end{abstract}

\section{Acknowledgments}

Not applicable.

\section{Authors' contributions}

SG and PS are chief investigators; they conceived the study, led the proposal and protocol development. GB, DP, and TZ drafted the manuscript. NV, DP, $G B, T Z, P S ̌$, and PŠ contributed to the protocol development. AZ, VJ, KR and AA helped with implementation. EŽ performs histological examination of the myocardium and contributed to drafting the manuscript. VS conceived and developed the statistical aspects of the study. GB, SG, DP, TZ, EŽ, AZ, VJ, NV, $\mathrm{KR}, \mathrm{AA}$, and PŠ will run the study day to day. All authors reviewed and approved the final version of the manuscript.

\section{Funding}

This study has received funding from European Social Fund (project No 09.3.3-LMT-K-712-01-0105) under grant agreement with the Research Council of Lithuania (LMTLT). The funder had no role in design of this study and will not have any role during its execution, analyses, interpretation of the data, or decision to submit results.

\section{Availability of data and materials}

Not applicable. Study patient enrollment and data collection is currently ongoing and no datasets were generated for analysis yet.

\section{Ethics approval and consent to participate}

The study (protocol, including qualitative and quantitative aspects, and trial materials, including patient information and consent form) was reviewed and approved by the Biomedical Research Ethics Committee of the Vilnius Region for VUH (No: 158200-18/9-1014-558) and by the North Denmark Region Committee on Health Research Ethics for AAUH (No: N-20180081). Written, informed consent to participate will be obtained from all study participants.

\section{Consent for publication}

Not applicable.

\section{Competing interests}

The authors declare that they have no competing interests.

\section{Author details}

${ }^{1}$ Clinic of Cardiac and Vascular Diseases, Institute of Clinical Medicine, Vilnius University Faculty of Medicine, Santariškiu str. 2, LT-08661 Vilnius, Lithuania. ${ }^{2}$ Department of Radiology, Nuclear Medicine and Medical Physics, Institute of Biomedical Sciences, Vilnius University Faculty of Medicine, Santariškiu str. 2, LT-08661 Vilnius, Lithuania. ${ }^{3}$ Department of Pathology, Forensic Medicine and Pharmacology, Institute of Biomedical Sciences, Vilnius University Faculty of Medicine, P. Baublio str. 5, LT-08406 Vilnius, Lithuania. Institute of Applied Mathematics, Vilnius University Faculty of Mathematics and Informatics, Naugarduko str. 24, LT-03225 Vilnius, Lithuania. ${ }^{5}$ Aalborg University Hospital, Clinical Institute of Aalborg University, Hobrovej 18-22, 9100 Aalborg, Denmark.

Received: 3 May 2020 Accepted: 24 May 2020

Published online: 08 June 2020

\section{References}

1. Chin CWL, Everett RJ, Kwiecinski J, Vesey AT, Yeung E, Esson G, et al. Myocardial fibrosis and cardiac decompensation in aortic stenosis. JACC Cardiovasc Imaging. 2017;10:1320-33.

2. Fielitz J, Hein S, Mitrovic V, Pregla R, Zurbrügg HR, Warnecke C, et al. Activation of the cardiac renin-angiotensin system and increased myocardial collagen expression in human aortic valve disease. J Am Coll Cardiol. 2001;37:1443-9.

3. Lee SP, Lee W, Lee JM, Park EA, Kim HK, Kim YJ, et al. Assessment of diffuse myocardial fibrosis by using MR imaging in asymptomatic patients with aortic stenosis. Radiology. 2015;274:359-69.

4. Balciunaite G, Skorniakov V, Rimkus A, Zaremba T, Palionis D, Valeviciene N, et al. Prevalence and prognostic value of late gadolinium enhancement on CMR in aortic stenosis: meta-analysis. Eur Radiol. 2020 Jan;30(1):640-51. https://doi.org/10.1007/s00330-019-06386-3.

5. Musa TA, Treibel TA, Vassiliou VS, Captur G, Singh A, Chin C, et al. Myocardial scar and mortality in severe aortic stenosis. Circulation. 2018 Oct 30;138(18):1935-47.

6. Treibel TA, Kozor R, Schofield R, Benedetti G, Fontana M, Bhuva AN, et al. Reverse myocardial remodeling following valve replacement in patients with aortic stenosis. J Am Coll Cardiol. 2018;71:860-71.

7. Everett RJ, Tastet L, Clavel MA, Chin CWL, Capoulade R, Vassiliou VS, et al. Progression of hypertrophy and myocardial fibrosis in aortic stenosis: a multicenter cardiac magnetic resonance study. Circ Cardiovasc Imaging. 2018;11:e007451.

8. Chin CW, Pawade TA, Newby DE, Dweck MR. Risk stratification in patients with aortic stenosis using novel imaging approaches. Circ Cardiovasc Imaging. 2015;8:e003421

9. Lee H, Park JB, Yoon YE, Park EA, Kim HK, Lee W, et al. Noncontrast myocardial T1 mapping by cardiac magnetic resonance predicts outcome in patients with aortic stenosis. JACC Cardiovasc Imaging. 2018;11:974-83.

10. Hwang IC, Kim HK, Park JB, Park EA, Lee W, Lee SP, et al. Aortic valve replacement-induced changes in native $\mathrm{T} 1$ are related to prognosis in severe aortic stenosis: $\mathrm{T} 1$ mapping cardiac magnetic resonance imaging study. Eur Heart J Cardiovasc Imaging. 2019 Aug 4. pii: jez201. doi: https:// doi.org/10.1093/ehjci/jez201.

11. Singh A, Horsfield MA, Bekele S, Khan JN, Greiser A, McCann GP. Myocardial $\mathrm{T} 1$ and extracellular volume fraction measurement in asymptomatic patients with aortic stenosis: reproducibility and comparison with age-matched controls. Eur Heart J Cardiovasc Imaging. 2015;16:763-70. 
12. Chin CW, Semple S, Malley T, White AC, Mirsadraee S, Weale PJ, et al. Optimization and comparison of myocardial T1 techniques at 3T in patients with aortic stenosis. Eur Heart J Cardiovasc Imaging. 2014;15:556-65.

13. Kockova R, Kacer P, Pirk J, Maly J, Sukupova L, Sikula V, et al. Native T1 relaxation time and extracellular volume fraction as accurate markers of diffuse myocardial fibrosis in heart valve disease- comparison with targeted left ventricular myocardial biopsy. Circ J. 2016;80:1202-9.

14. Child N, Suna G, Dabir D, Yap ML, Rogers T, Kathirgamanathan M, et al. Comparison of MOLLI, shMOLLLI, and SASHA in discrimination between health and disease and relationship with histologically derived collagen volume fraction. Eur Heart J Cardiovasc Imaging. 2018;19:768-76.

15. Everett RJ, Treibel TA, Fukui M, Lee H, Rigolli M, Singh A, et al. Extracellular myocardial volume in patients with aortic stenosis. J Am Coll Cardiol. 2020 Jan 28;75(3):304-16. https://doi.org/10.1016/j.jacc.2019.11.032.

16. Nagata Y, Takeuchi M, Wu VC, Izumo M, Suzuki K, Sato K, et al. Prognostic value of $L V$ deformation parameters using $2 \mathrm{D}$ and $3 \mathrm{D}$ speckle-tracking echocardiography in asymptomatic patients with severe aortic stenosis and preserved LV ejection fraction. J Am Coll Cardiol Img. 2015;8:235-45.

17. Park SJ, Cho SW, Kim SM, Ahn J, Carriere K, Jeong DS, et al. Assessment of myocardial fibrosis using multimodality imaging in severe aortic stenosis: comparison with histologic fibrosis. JACC Cardiovasc Imaging. 2019 Jan; 12(1):109-19. https://doi.org/10.1016/j.jcmg.2018.05.028.

18. Harris PA, Taylor R, Thielke R, Payne J, Gonzalez N, Conde JG. Research electronic data capture (REDCap)-a metadata-driven methodology and workflow process for providing translational research informatics support. J Biomed Inform. 2009;42(2):377-81. https://doi.org/10.1016/j.jbi.2008.08.010.

19. Baumgartner H, Falk V, Bax JJ, De Bonis M, Hamm C, Holm PJ, et al. ESC scientific document group. 2017 ESC/EACTS guidelines for the management of valvular heart disease. Eur Heart J. 2017 Sep 21;38(36):2739-91. https:// doi.org/10.1093/eurheartj/ehx391.

20. Nashef SA, Roques F, Sharples LD, Nilsson J, Smith C, Goldstone AR, et al. EuroSCORE II. Eur J Cardiothorac Surg. 2012:41:734-45.

21. Shih T, Paone G, Theurer PF, McDonald D, Shahian DM, Prager RL. The society of thoracic surgeons adult cardiac surgery database version 2.73: more is better. Ann Thorac Surg. 2015;100:516-21.

22. Balan $\mathrm{P}$, Zhao Y, Johnson S, Arain S, Dhoble A, Estrera A, et al. The Society of Thoracic Surgery Risk Score as a predictor of 30-day mortality in Transcatheter vs surgical aortic valve replacement: A single-center experience and its implications for the development of a TAVR riskprediction model. J Invasive Cardiol. 2017 Mar;29(3):109-14.

23. de Arenaza DP, Pepper J, Lees B, Rubinstein F, Nugara F, Roughton M, et al. Preoperative 6-minute walk test adds prognostic information to Euroscore in patients undergoing aortic valve replacement. Heart. 2010;96:113-7.

24. Rector TS, Cohn JN. Assessment of patient outcome with the Minnesota living with heart failure questionnaire: reliability and validity during a randomized, double-blind, placebo-controlled trial of pimobendan. Pimobendan multicenter research group. Am Heart J. 1992;124:1017-25.

25. Supino PG, Borer JS, Franciosa JA, Preibisz JJ, Hochreiter C, Isom OW, et al. Acceptability and psychometric properties of the Minnesota living with heart failure questionnaire among patients undergoing heart valve surgery: validation and comparison with SF-36. J Card Fail. 2009;15:267-77.

26. Lang RM, Badano LP, Mor-Avi V, Afilalo J, Armstrong A, Ernande L, et al. Recommendations for cardiac chamber quantification by echocardiography in adults: an update from the American Society of Echocardiography and the European Association of Cardiovascular Imaging. J Am Soc Echocardiogr. 2015;28:1-39.

27. Baumgartner H, Hung J, Bermejo J, Chambers JB, Edvardsen T, Goldstein S, et al. Recommendations on the echocardiographic assessment of aortic valve stenosis: a focused update from the European Association of Cardiovascular Imaging and the American Society of Echocardiography. Eur Heart J Cardiovasc Imaging. 2017;18:254-75.

28. Nagueh SF, Smiseth OA, Appleton CP, Byrd BF 3rd, Dokainish H, Edvardsen $T$, et al. Recommendations for the evaluation of left ventricular diastolic function by echocardiography: an update from the American Society of Echocardiography and the European Association of Cardiovascular Imaging. Eur Heart J Cardiovasc Imaging. 2016;17:1321-60

29. Voigt JU, Pedrizzetti G, Lysyansky P, Marwick TH, Houle H, Baumann R, et al. Definitions for a common standard for 2D speckle tracking echocardiography: consensus document of the EACVI/ASE/industry task force to standardize deformation imaging. Eur Heart J Cardiovasc Imaging. 2015:16:1-11.
30. Taylor AJ, Salerno M, Dharmakumar R, Jerosch-Herold M. T1 mapping: basic techniques and clinical applications. JACC Cardiovasc Imaging. 2016;9:6781.

31. de Meester de Ravenstein C, Bouzin C, Lazam S, Boulif J, Amzulescu M, Melchior J, et al. Histological validation of measurement of diffuse interstitial myocardial fibrosis by myocardial extravascular volume fraction from modified look-locker imaging (MOLLI) T1 mapping at 3 T. J Cardiovasc Magn Reson. 2015;17:48.

32. Puntmann VO, Voigt T, Chen Z, Mayr M, Karim R, Rhode $K$, et al. Native T1 mapping in differentiation of normal myocardium from diffuse disease in hypertrophic and dilated cardiomyopathy. J Am Coll Cardiol Img. 2013;6: 475-84.

33. Ugander M, Oki AJ, Hsu LY, Kellman P, Greiser A, Aletras AH, et al. Extracellular volume imaging by magnetic resonance imaging provides insights into overt and sub-clinical myocardial pathology. Eur Heart J. 2012; 33:1268-78

34. Kappetein AP, Head SJ, Généreux P, Piazza N, van Mieghem NM, Blackstone $\mathrm{EH}$, et al. Updated standardized endpoint definitions for transcatheter aortic valve implantation: the valve academic research Consortium-2 consensus document (VARC-2). Eur J Cardiothorac Surg. 2012;42:S45-60.

35. Akins CW, Miller DC, Turina MI, Kouchoukos NT, Blackstone EH, Grunkemeier $G L$, et al. Guidelines for reporting mortality and morbidity after cardiac valve interventions. J Thorac Cardiovasc Surg. 2008;135:732-8.

36. Chan AW, Tetzlaff JM, Altman DG, Laupacis A, Gøtzsche PC, Krle A-Jerić K, et al. SPIRIT 2013 statement: defining standard protocol items for clinical trials. Rev Panam Salud Publica. 2015 Dec;38(6):506-14

37. R Core Team. R: A language and environment for statistical computing. $R$ Foundation for Statistical Computing, Vienna, Austria. 2018. https://www.Rproject.org/.

38. Qiu W, Chavarro J, Lazarus R. Rosner B. PowerSurvEpi: Ma J. R package for power and sample size calculation for survival analysis of epidemiological studies; 2018. https://cran.r-project.org/web/packages/powerSurvEpi/index. html).

\section{Publisher's Note}

Springer Nature remains neutral with regard to jurisdictional claims in published maps and institutional affiliations.
Ready to submit your research? Choose BMC and benefit from:

- fast, convenient online submission

- thorough peer review by experienced researchers in your field

- rapid publication on acceptance

- support for research data, including large and complex data types

- gold Open Access which fosters wider collaboration and increased citations

- maximum visibility for your research: over $100 \mathrm{M}$ website views per year

At BMC, research is always in progress.

Learn more biomedcentral.com/submissions 